\title{
Cartographie et caractérisation floristique de la forêt marécageuse de Lokoli (Bénin)
}

\author{
A.C. ADOMOU ${ }^{1 *}$, A. MAMA ${ }^{2}$, R. MISSIKPODE ${ }^{2}$ et B. SINSIN $^{2}$ \\ ${ }^{1}$ Département de Biologie Végétale, Faculté des Sciences et Techniques, Université d'Abomey-Calavi, \\ 01 BP 4521 Cotonou, Bénin. \\ ${ }^{2}$ Laboratoire d'Ecologie Appliquée, Faculté des Sciences Agronomiques, Université d'Abomey-Calavi, \\ 01 BP 526 Cotonou, Bénin. \\ *Auteur correspondant,Email: adomouaristide@yahoo.fr,adomou.a@gmail.com
}

\section{RESUME}

La présente étude s'inscrit dans le cadre de l'évaluation des ressources biologiques de la forêt marécageuse de Lokoli en république du Bénin en vue de définir les stratégies pour sa conservation durable. Le SIG a permis de réaliser la carte de végétation de la forêt qui a servi de base pour la collecte de données floristiques et dendrométriques. La surface d'inventaire des placeaux est de $30 \times 30 \mathrm{~m}$. Au total, 125 espèces végétales sont recensées dont 30 espèces menacées de disparition au Bénin. La densité spécifique totale est de 53 espèces/ha et celle des individus de $\mathrm{dbh} \geq 10 \mathrm{~cm}$ est d'environ 15 espèces/ha. L'indice de diversité de Shannon $\left(\mathrm{H}^{\prime}=3,24\right.$ bits) est moyen alors que l'équitabilité de Pielou $(\mathrm{Eq}=0,63)$ est faible. Les espèces les plus dominantes écologiquement sont: Alstonia congensis, Xylopia rubescens, Syzygium owariense et Raphia hookeri. Les principales formes de pressions sont: coupe de bois, collecte de plantes médicinales et l'exploitation du raphia. Cependant, la forêt apparaît bien préservée avec environ $78 \%$ de couverture forestière. Trois principaux groupements végétaux sont décrits et la densité de raphia est proportionnelle au degré de dégradation des divers faciès végétaux.

(c) 2009 International Formulae Group. All rights reserved.

Mots clés: Bénin, forêt marécageuse de Lokoli, SIG, diversité floristique, conservation, Dahomey Gap.

\section{INTRODUCTION}

La végétation du Sud Bénin est fortement influencée par le phénomène énigmatique du "Dahomey Gap" qui est une interruption de la ceinture forestière devant relier les deux bastions forestiers de l'Afrique centrale et de l'Afrique occidentale. Ainsi, la forêt dense humide n'existe que sous forme de petits îlots (moins de 0,50 à quelques milliers d'hectares) et l'unité sempervirente de terre ferme y est complètement absente. Dans ce couloir de sécheresse, les zones humides abritent une gamme variée d'écosystèmes forestiers dont certains constitueraient des micro-refuges pour les espèces animales et végétales rares (PAZH, 1997). Malheureu- sement, le couvert végétal des zones humides a été considérablement modifié depuis le début du processus du peuplement des zones humides, suite à la croissance effrénée de la population. Cependant, il subsiste de rares lambeaux de forêt marécageuse et riveraine qu'il faudra conserver car ils constituent des réservoirs d'espèces rares. Dans le souci de préserver ces écosystèmes fragiles, un programme d'aménagement, de bio-monitoring et de gestion durable des forêts reliques des zones humides a été initié par le PAZH (1997). La réussite d'un tel programme nécessite une connaissance approfondie des caractéristiques fauniques, floristiques et structurales de ces écosystèmes. 
Plusieurs études floristiques ont été menées sur la végétation des zones humides du Sud Bénin parmi lesquelles celles de Mondjannagni (1969), Paradis (1975), Sokpon et al. (2001). La forêt communautaire de Lokoli demeure l'unique écosystème marécageux le mieux préservé au Bénin et représente le seul témoin qui nous renseigne sur la sylve ancienne. Elle est restée inconnue du monde scientifique jusque dans les années 2000 et n'a pratiquement pas fait l'objet d'inventaire botanique. La cartographie de la végétation est aussi envisagée afin de doter les différents acteurs (décideurs et gestionnaires) des outils de base pouvant contribuer à une gestion durable de cet écosystème.

La présente étude s'inscrit dans le cadre $\mathrm{du}$ processus de l'aménagement de la forêt marécageuse de Lokoli où les inventaires fauniques ont révélé l'existence d'espèces endémiques, menacées ou rares (Sinsin et Assogbadjo, 2002). Elle vise à : réaliser la carte de végétation de la forêt, caractériser les groupements végétaux et déterminer la structure démographique des espèces ligneuses les plus exploitées.

\section{MATERIEL ET METHODES Milieu d'étude}

La forêt marécageuse de Lokoli est située dans l'arrondissement de Koussoukpa, commune de Zogbodomey, située dans le département du Zou (Bénin) au point de latitude $7^{\circ} 03^{\prime} \mathrm{N}$ et longitude $2^{\circ} 15^{\prime} \mathrm{E}$ (figure 1). D'une superficie d'environ 3000 ha, elle est située dans la zone guinéenne. Elle est localisée dans la partie nord de la basse vallée de l'Ouémé. Le régime pluviométrique est bimodal avec une pluviométrie annuelle de $1112 \mathrm{~mm}$ d'eau répartie sur deux saisons.

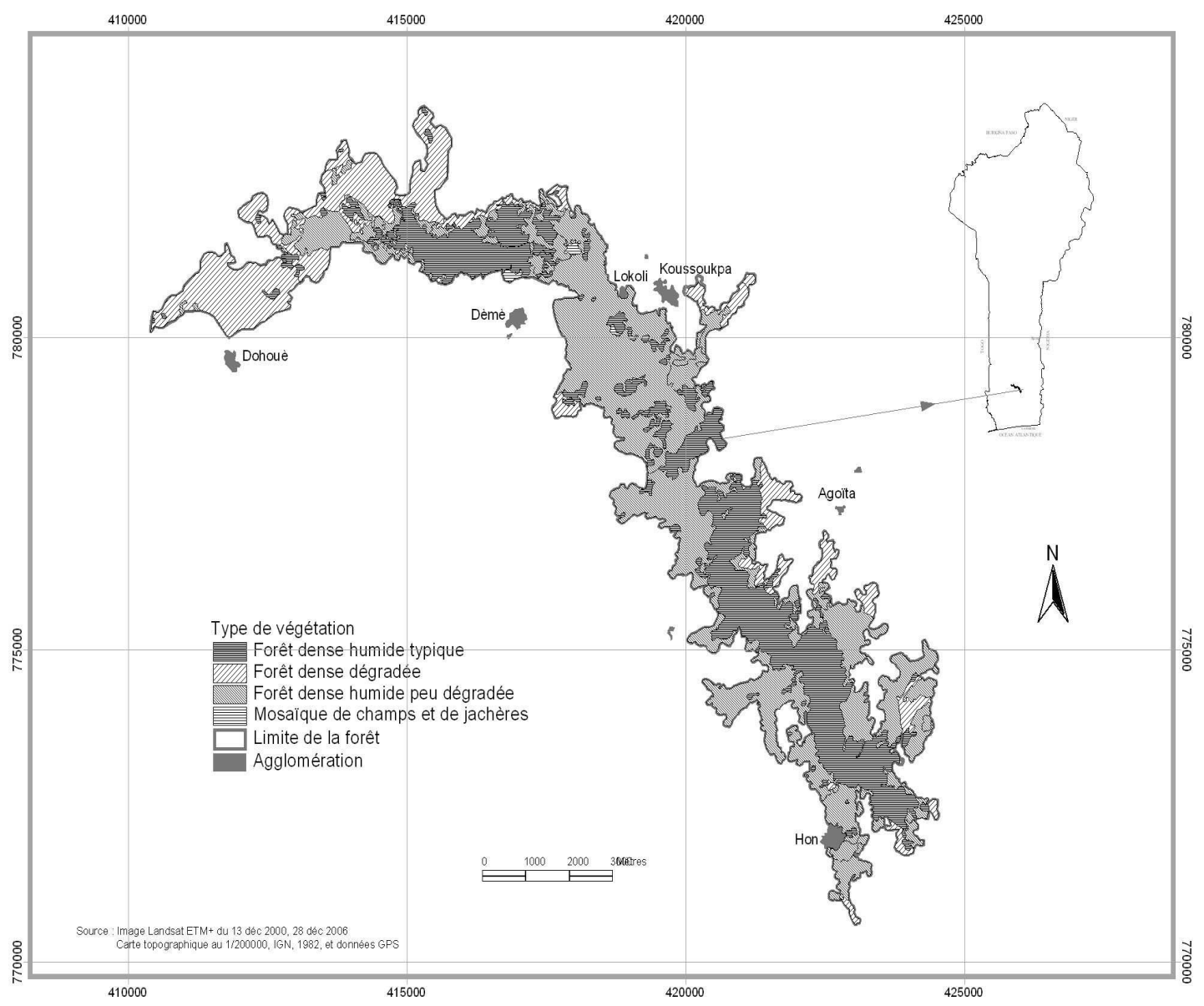

Figure 1: Carte de végétation de la forêt marécageuse de Lokoli montrant son état de conservation. 
La température moyenne annuelle est de 26$28{ }^{\circ} \mathrm{C}$. L'amplitude thermique est de $9,7^{\circ} \mathrm{C}$. Le sol est argilo-limoneux et tourbeux.

La forêt est entourée de trois villages (Lokoli, Koussoukpa et Dèmè) avec une population de 4810 habitants (INSAE, 2002). Les principales activités sont l'agriculture, l'artisanat, le ramassage d'escargots, l'exploitation du vin et des feuilles de Raphia hookeri.

\section{Méthodes}

La cartographie de la végétation sur la base du SIG a consisté à: l'interprétation visuelle des images Landsat ETM+ de 2000 et décembre 2006, la classification "supervisée", le "contrôle terrain" à partir de l'image classifiée, l'exportation de la classification dans ArcView GIS 3.3 suivie de la digitalisation des différentes classes de végétation. La classification "supervisée" est réalisée par la logique floue et les objets sont classifiés en utilisant les techniques de "plus proches voisins" ainsi que des fonctions statistiques d'appartenance (Ourab et al., 2003 ; Palatucci et Mitchell, 2007).

Les points d'inventaire floristique sont ceux dont le recouvrement arborescent est d'au moins $70 \%$ c'est-à-dire les faciès de forêt dense humide typique (figure 1). Les relevés phytosociologiques sont effectués dans des placeaux de 30 x $30 \mathrm{~m}$ selon la méthode classique de Braun-Blanquet (1932); 26 relevés floristiques sont réalisés. Le diamètre à hauteur d'homme $(\mathrm{dbh} \geq 10 \mathrm{~cm})$ à $1,30 \mathrm{~m} \mathrm{du}$ sol ou à $30 \mathrm{~cm}$ au-dessus des contreforts des ligneux est mesuré à l'aide d'un ruban pi. Les coordonnées géographiques des placettes sont prises à l'aide d'un GPS (Global Positioning System). Les espèces sont identifiées à l'aide de la Flore Analytique du Bénin (Akoègninou et al., 2006) et le Guide de terrain des ligneux des forêts denses humides de l'Afrique de l'Ouest (Hawthorne et Jongkind, 2006).

Une enquête ethnobotanique rapide a permis d'apprécier l'importance de la forêt pour la population locale et de recenser les diverses formes de pression sur elle.

La classification hiérarchique ascendante utilisant "cluster analysis" (méthode de Ward) a permis de faire la typologie des groupements végétaux.

La diversité floristique a été appréciée en utilisant un certain nombre d'indices et de variables présentés ci-dessous.

L'indice de Shannon (Shannon, 1948), $H^{\prime}$ :

$\mathrm{H}^{\prime}=-\sum_{i=1}^{s} p_{i} \log _{2} p_{i}$

avec $p_{i}=$ l'abondance relative de l'espèce $i$.

Cet indice intègre à la fois la richesse spécifique et l'abondance relative des espèces. Il est utile car sa valeur augmente non seulement en fonction du nombre d'espèces, mais aussi selon l'abondance relative de chaque espèce dans la communauté. L'indice est maximal quand tous les individus sont répartis d'une façon égale sur toutes les espèces (Frontier, 1983).

L'indice d'équitabilité de Pielou

$\mathrm{J}=\mathrm{H}^{\prime} / \log _{2} \mathrm{~S}$

avec $\mathrm{S}=$ la richesse spécifique.

L'indice d'équitabilité ou de régularité exprime, dans un échantillon donné, le rapport de la diversité atteinte $\left(\mathrm{H}^{\prime}\right)$ et la diversité maximale $\left(\log _{2} \mathrm{~S}\right)$ pouvant être obtenue avec le même nombre de taxons (Frontier et PichodViale, 1991). Cet indice varie de 0 à 1 ; il est maximal quand les espèces ont des abondances identiques dans le peuplement et il est minimal quand une seule espèce domine tout le peuplement.

La valeur d'importance d'une espèce (IVI) est la somme de sa fréquence relative (fr), de sa densité relative (dr) et de sa dominance relative (domr). La fréquence relative d'une espèce est exprimée en termes de proportion de la somme des fréquences de toutes les espèces. La densité d'une espèce est le nombre d'individus par hectare (tige/ ha). La dominance est exprimée en termes d'aire basale $\left(\Pi / 4 \times \mathrm{D}^{2}, \mathrm{D}=\mathrm{dbh}\right.$ et $\left.\Pi=3,14\right)$.

La valeur d'importance d'une famille (FIV) est la somme de sa densité relative (dr), de sa dominance relative (domr) et de sa diversité relative (divr). Les variables 'dr' et 'domr' sont calculées en considérant les espèces appartenant à la même famille botanique. La diversité est exprimée en termes du nombre d'espèces par famille.

IVI et FVI sont des indices quantitatifs permettant d'identifier les espèces et familles écologiquement importantes dans une communauté végétale (Curtis et Macintosh, 1950; Mori et Boom, 1987). IVI et FIV varie de 0 (absence de dominance) à 300 (mono- 
dominance). Selon Reitsma (1988), les espèces et familles écologiquement importantes sont celles ayant une Valeur d'Importance IV > 10.

Pour rendre compte de la structure démographique du peuplement ligneux et de quelques espèces ligneuses exploitées par la population, la répartition des individus par classe de diamètre est effectuée.

\section{RESULTATS}

Cartographie et typologie des faciès de végétation

La cartographie de la forêt marécageuse de Lokoli a permis de délimiter la forêt et de préciser sa superficie qui est estimée à 2964,96 ha. Quatre faciès de végétation peuvent être distingués (Figure 1) :

* La forêt dense typique: elle occupe $30,8 \%$ de la superficie totale, le recouvrement arborescent est d'au moins $70 \%$.

* La forêt dense peu dégradée: elle représente $47,6 \%$ de la superficie totale, le recouvrement arborescent est de 10-30\%.
* La forêt dense dégradée: elle représente $21,2 \%$ de la superficie totale, le recouvrement arborescent reste inférieur à $5 \%$.

* Les Mosaïques de champs et jachères: elles ne représentent que $0,4 \%$ de la superficie totale de la forêt.

Les relevés phytosociologiques effectués dans les faciès de forêt dense typique et peu dégradée ont permis de caractériser floristiquement la végétation naturelle de l'écosystème marécageux de Lokoli.

\section{Caractérisation des groupements végétaux}

La classification hiérarchique ascendante de 26 relevés floristiques et 126 espèces a permis de discriminer (à $60 \%$ de dissimilarité) deux grands groupes de relevés en fonction de l'état de dégradation (Figure 2): (1) Groupe de relevés effectués dans les faciès de forêt dense humide typique (G1) où le recouvrement arborescent est supérieur à $70 \%$; deux sous-groupes se distinguent :

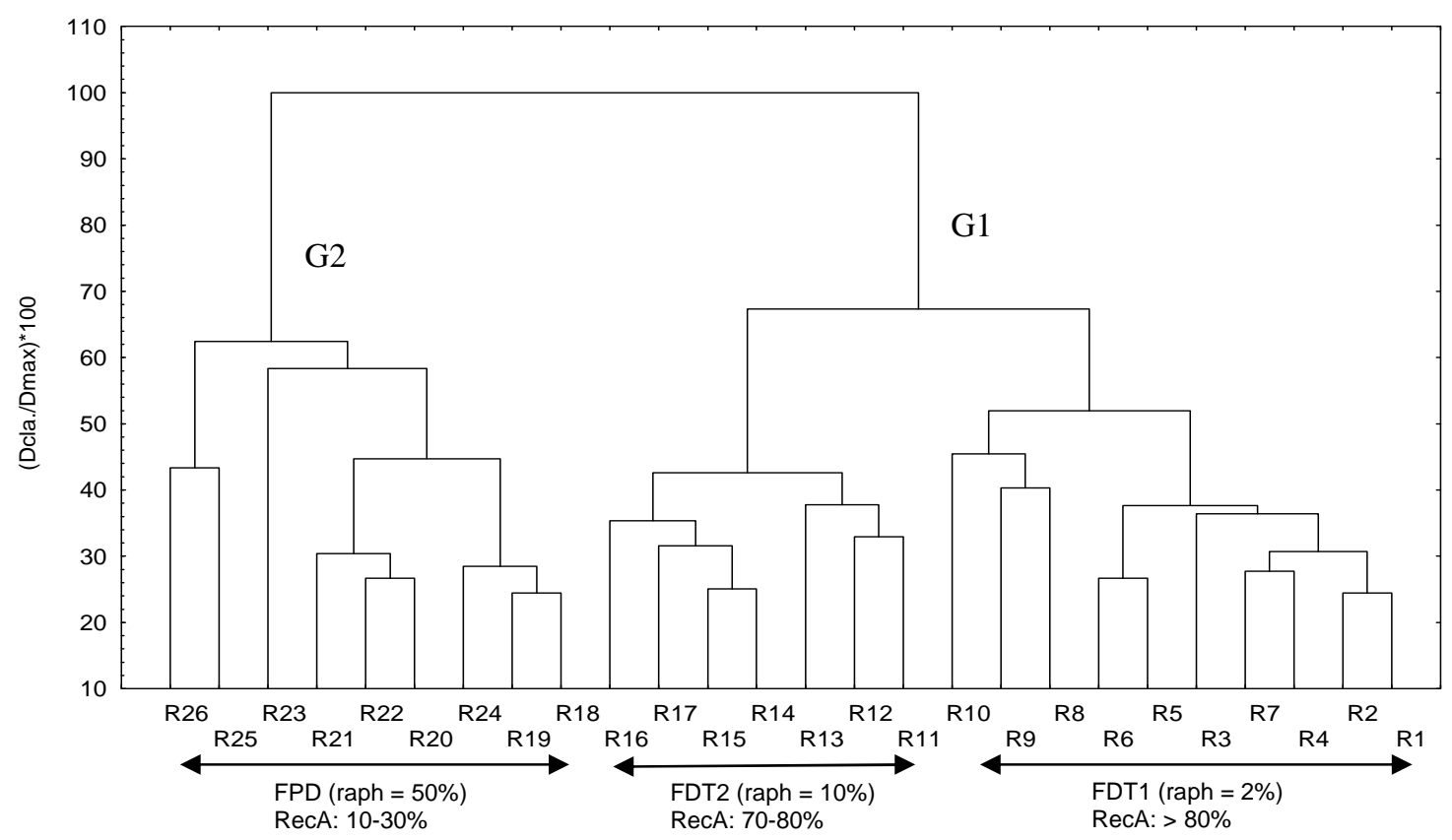

Figure 2: Dendrogramme montrant les différents groupements végétaux. FPD: Forêt dense peu dégradée, FDT1: Forêt dense typique avec $2 \%$ de raphia, FDT2: Forêt dense typique avec $10 \%$ de raphia; raph: densité relative de Raphia hookeri, RecA: Recouvrement arborescent. Arbre de 26 variables, Méth. de Ward, Dist. Euclidiennes. 
le faciès à Alstonia congensis (FDT1) où la densité relative de Raphia hookeri est de 2\% et le faciès à Hallea ledermannii (FDT2) où la densité relative de $R$. hookeri est de $10 \%$. Le faciès FDT1 est caractérisé par un recouvrement arborescent supérieur à $80 \%$ avec de petites trouées qui sont colonisées par Raphia hookeri. Le faciès FDT2 est caractérisé par un recouvrement arborescent de $70-80 \%$ avec des trouées plus larges.

(2) Groupe de relevés effectués dans les faciès de forêt peu dégradée (G2) ayant un recouvrement arborescent de 10-30\%. Il est caractérisé par une forte représentation de Raphia hookeri dont la densité relative est de $50 \%$. Les espèces comme Ficus congensis, Ficus vogeliana, Anthocleista vogelii et Macaranga heudelotii y sont abondantes. On observe aussi une forte représentation des espèces typiques de milieu marécageux dont la diversité relative est estimée à $48 \%$ de la richesse spécifique totale (125 espèces). On note la présence des espèces comme Uapaca paludosa, Anthostema aubryanum, Homalium africanum et Rothmannia munsae qui sont des espèces caractéristiques des milieux fortement hydromorphes.

\section{Structure forestière}

Le profil structural de la forêt montre 4 strates. La strate arborescente supérieure, de 15 à $30 \mathrm{~m}$, est dominée par Alstonia congensis, Xylopia rubescens, Syzygium owariense et Hallea ledermannii. La strate arborescente inférieure, de 8-15 m, comprend Spondianthus preussii, Anthocleista vogelii et Nauclea diderrichii. La strate arbustive, de 3$8 \mathrm{~m}$, est dominée par Pterocarpus santalinoides, Ficus vogeliana et Psychotria articulata. Enfin, la strate herbacée, de 0-3 m de hauteur, comporte les espèces telles que Lasiomorpha senegalensis, Cyclosorus striatus, Nephrolepis bisserata, Tacateza pedicellata, Grewia barombiensis et Lycopodium cernuum.

La densité des ligneux de $\mathrm{dbh} \geq 10 \mathrm{~cm}$ est de 620 tiges/ha; les valeurs restent similaires d'un groupement à un autre. Les espèces les plus représentées en termes de densité sont Alstonia congensis (24\%) et Xylopia rubescens $(22 \%)$.

L'aire basale évaluée pour l'ensemble de la forêt est de $44,9 \mathrm{~m}^{2} / \mathrm{ha}$; les espèces telles que Alstonia congensis (35\%) et Xylopia rubescens (24\%) y contribuent fortement. L'estimation pour les groupements végétaux pris séparément montre qu'elle décroît avec le degré de dégradation : $43 \mathrm{~m}^{2} /$ ha pour les forêts denses typiques et $21 \mathrm{~m}^{2} /$ ha pour la forêt dense peu dégradée.

La structure diamétrique (Figure 3) du peuplement ligneux des divers groupements végétaux décrits montre une décroissance régulière de la fréquence relative des faibles classes de diamètre aux plus fortes. Deux types de tendance sont observés :

* Ajustement linéaire (forêts typiques, figure 3ab) : l'importance des faibles classes de diamètres $(10-30 \mathrm{~cm})$ est de $63 \%$ en moyenne ; celle des fortes classes de diamètre $(\geq 40 \mathrm{~cm}$ ) est de $19 \%$.

* Ajustement logarithmique (forêt dense peu dégradée, figure 3c) : la proportion des faibles classes de diamètres $(10-30 \mathrm{~cm})$ est de $89 \%$ en moyenne ; celle des fortes classes $(40-50 \mathrm{~cm})$ est très faible $(2,35 \%)$ avec une absence totale des individus de diamètre $\geq 50 \mathrm{~cm}$.

\section{Biodiversité végétale}

Au total 125 espèces, appartenant à 106 genres et 55 familles, sont recensées. Les familles les plus riches en espèces sont: Rubiaceae (17 espèces soit 14\%), Leguminosae (10 espèces soit $8 \%)$ et Moraceae ( 9 espèces, soit $9 \%$ ).

La richesse spécifique des individus de $\mathrm{dbh} \geq 10 \mathrm{~cm}$ est de 35 espèces groupées en 28 genres et 18 familles. L'indice de Shannon $\left(\mathrm{H}^{\prime}=3,24\right.$ bits) est moyen alors que l'équitabilité de Pielou $(\mathrm{Eq}=0,63)$ est faible ; les valeurs restent similaires d'un groupement à un autre.

Les valeurs d'importance des espèces et des familles montrent que 8 espèces végétales et 7 familles botaniques sont écologiquement dominantes (Tableaux 1 et 2). Les espèces les plus importantes écologiquement dominantes sont Alstonia congensis (IVI $=69,79)$ et Xylopia rubescens (56,70; Tableau 1). Les familles ayant les plus fortes valeurs d'importance sont les Annonaceae et les Apocynaceae (Tableau 2). 

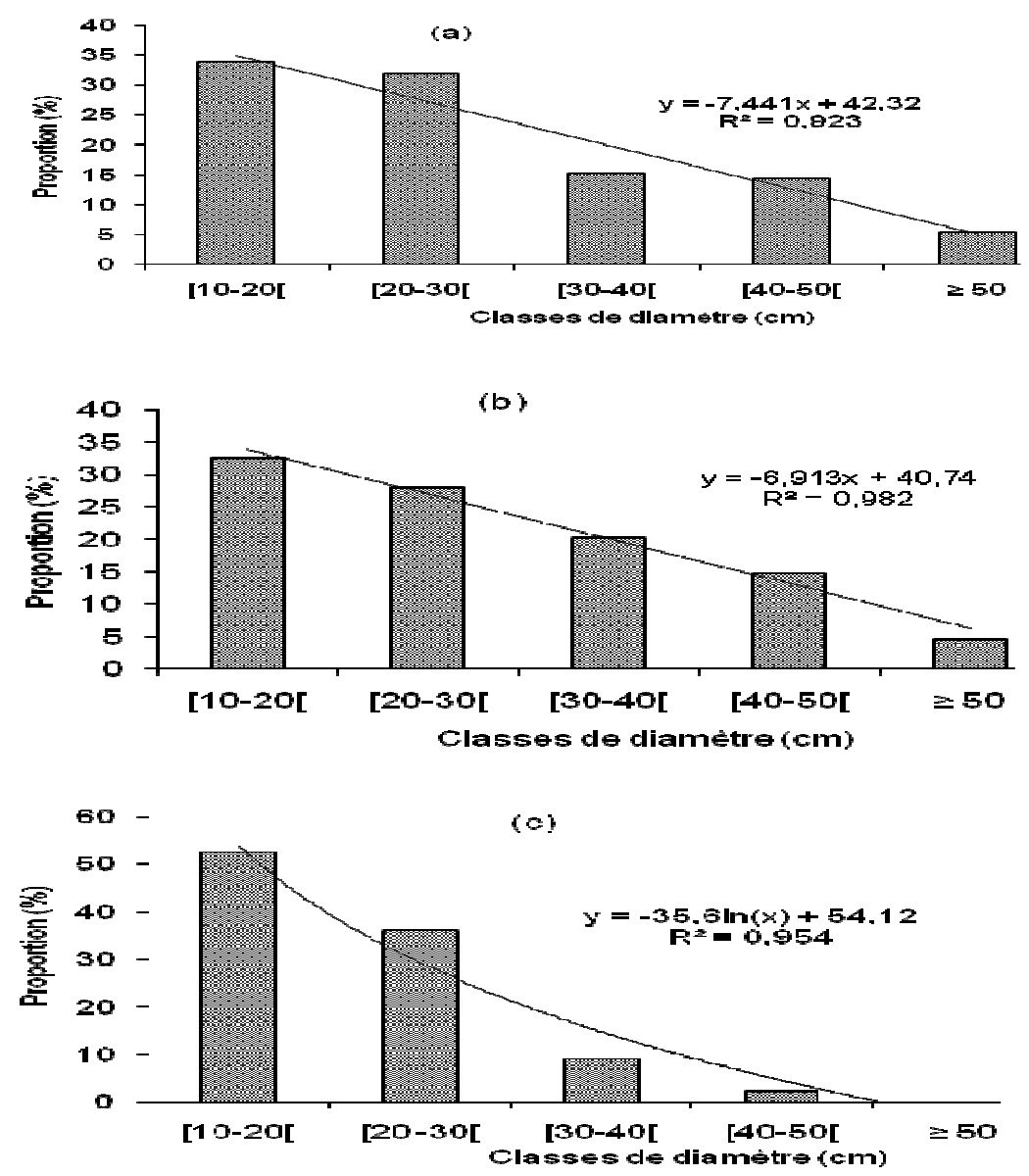

Figure 3 : Structure diamétrique du peuplement arborescent des groupements végétaux de la forêt marécageuse de Lokoli. (a) : Forêt dense typique à $2 \%$ de raphia (FDT1, 344 individus de tiges recensés), (b) : Forêt dense typique à 10\% de raphia (FDT2, 243 tiges), (c) : Forêt dense peu dégradée (FPD, 255 tiges).

Tableau 1: Valeurs d'importance des espèces (IVI).

\begin{tabular}{lcccc}
\hline \multicolumn{1}{c}{ Espèces } & Dr $(\boldsymbol{\%})$ & Fr $(\%)$ & Domr $(\%)$ & IVI \\
\hline Alstonia congensis & 24,16 & 10,0402 & 35,5929 & 69,7931 \\
Xylopia rubescens & 22,16 & 10,0401 & 24,5008 & 56,7009 \\
Raphia hookeri & 17,2 & 8,4337 & 8,5753 & 34,2090 \\
Syzygium owariense & 5,99 & 8,0321 & 13,4977 & 27,5198 \\
Spondianthus preussii & 8,67 & 9,6385 & 2,9178 & 21,2263 \\
Hallea ledermannii & 4,75 & 7,6305 & 4,4992 & 16,8797 \\
Cleistopholis patens & 3,85 & 7,2289 & 0,9132 & 11,9921 \\
Nauclea diderrichii & 1,31 & 5,6225 & 4,3879 & 11,3204 \\
Autres & 5,31 & 19,2773 & 2,6050 & 50,3587 \\
\hline Total & 100 & 100 & 100 & 300 \\
\hline
\end{tabular}

$\mathrm{Dr}=$ Densité relative; $\mathrm{Fr}=$ Fréquence relative; Domr $=$ Dominance relative $)$. 
Tableau 2: Valeurs d'importance des familles (FIV).

\begin{tabular}{lcccc}
\hline \multicolumn{1}{c}{ Famille } & Divr $\mathbf{( \% )}$ & Dr (\%) & Domr (\%) & FIV \\
\hline Annonaceae & 11,76 & 26,22 & 25,4648 & 63,4448 \\
Apocynaceae & 2,94 & 24,16 & 35,5929 & 62,6929 \\
Rubiaceae & 17,66 & 7,29 & 9,7969 & 34,7469 \\
Arecaceae & 5,89 & 17,27 & 8,6049 & 31,7649 \\
Euphorbiaceae & 11,76 & 9,71 & 3,3413 & 24,8113 \\
Myrtaceae & 2,94 & 5,99 & 13,4977 & 22,4277 \\
Moraceae & 11,76 & 4,81 & 2,3134 & 18,8834 \\
Autres & 23,52 & 1,38 & 0,7238 & 41,2281 \\
\hline Total & 100 & 100 & 100 & 300 \\
\hline
\end{tabular}

$\overline{\text { Divr }=\text { Diversité relative; } \text { Dr }=\text { Densité relative; Fr }=\text { Fréquence relative; Domr = Dominance relative }}$.

\section{Pression anthropique}

Les espèces les plus fréquemment prélevées et leurs usages sont récapitulés dans le tableau 3. La forêt constitue pour la population riveraine une source de produits forestiers ligneux et non-ligneux. Les espèces telles que Alstonia congensis et Hallea ledermanii fournissent du bois d'œuvre et de service très recherché par la population locale. Mais, Raphia hookeri reste l'espèce la plus prisée car fournissant une gamme variée de matières premières dont le stipe et les feuilles. L'écorce des espèces telles que Nauclea diderrichii, Syzygium owariense et Ficus trichopoda est médicinale, très exploitée et commercialisée.

Les individus de dbh compris entre 10 et $20 \mathrm{~cm}$ sont très sollicités pour la réalisation des charpentes, des plafonds et des greniers. Il s'agit des espèces comme Psychotria articulata qui est un arbuste et les jeunes pieds d'arbres tels que Alstonia congensis, Xylopia rubescens Syzygium owariense et Spondianthus preussii. Il est à noter que les espèces utiles comme Hallea ledermannii $(7,6 \%)$, Spondianthus preussii $(9,6 \%)$ et Psychotria articulata $(11,54 \%)$ affichent de faibles valeurs de fréquence. Sept espèces végétales, soit $5,8 \%$ de la flore, sont identifiées comme étant de grande valeur médicinale pour la population locale (Tableau $3)$.

La structure démographique des espèces ligneuses prisées (Figure 4) montre une bonne représentation des juvéniles $(10 \leq \mathrm{dbh}<20$ $\mathrm{cm})$ avec des fréquences relatives variant entre 31 et $71 \%$. Les différentes distributions par classes de diamètre peuvent être regroupées en trois types:
* Type 1 chez Ficus trichopoda et Syzygium owariense: tendance vers la distribution en "L" avec un bon recrutement des classes de diamètre $20-40 \mathrm{~cm}$ chez $S$. owariense et 30 à $40 \mathrm{~cm}$ chez $F$. trichopoda.

* Type 2 chez Nauclea diderrichii et Spondianthus preussii: tendance vers la distribution en "L" avec une absence de certaines classes de diamètre.

* Type 3 chez Alstonia congensis et Hallea stipulosa : distribution erratique traduisant une répartition diamétrique irrégulière des individus.

\section{DISCUSSION}

Sur le plan phytosociologique, on note une importance des espèces de l'ordre des Mytragyno-Raphietalia (Lebrun et Gilbert, 1954), en occurrence Alstonia congensis, Hallea ledermannii, Raphia hookeri, Cyclosorus striatus et Ficus trichopoda. Néanmoins, quelques espèces de nos relevés figurent sur la liste des espèces caractéristiques de l'ordre des Uapacetalia de Mangenot (1956), il s'agit de: Funtumia africana, Hallea ledermannii, Spondianthus preussii et Uapaca paludosa. La présence de Hallea ledermannii et Spondianthus preussii détermine la variante paludosum de l'association Diospyro-Mapanietum, caractéristique des bas-fonds. La végétation de la forêt de Lokoli correspond au groupement à Alstonia congensis et Psychotria articulata de Akoègninou (2004) qui est un faciès de dégradation du groupement à Hallea ledermannii et Lasiomorpha senegalensis de Adomou et al. (2007). 
Tableau 3: Usages et importance de quelques espèces dans la forêt de Lokoli.

\begin{tabular}{|c|c|c|c|c|c|c|}
\hline Espèces & Familles & $\begin{array}{c}\mathbf{G} \\
\left(\mathbf{m}^{2} / \mathbf{h a}\right)\end{array}$ & $\operatorname{fr}(\%)$ & $\begin{array}{c}\text { Organes } \\
\text { utilisé }\end{array}$ & Usage & Pression \\
\hline Aframomum sceptrum & Zingiberaceae & - & 3,85 & Rhizomes & Alimentaire, épices & + \\
\hline Agelaea pentagyna & Connaraceae & - & 42,31 & Feuilles & Médicinal: fragilité de la fontanelle chez le nouveau-né & + \\
\hline Alstonia congensis & Apocynaceae & 15,98 & 100 & Bois & $\begin{array}{l}\text { Bois d'œuvre et de service (Pirogue, cercueil, plafond, } \\
\text { charpente) }\end{array}$ & +++ \\
\hline Aphania senegalensis & Sapindaceae & 0,032 & 4 & Tiges & fabrication des entailles pour fixer les tam-tams & + \\
\hline Cleistopholis patens & Annonaceae & 0,41 & 69,23 & Ecorces & Aromatique pour embaumer le vin de Raphia gâté & + \\
\hline Culcasia scandens & Araceae & - & 42,31 & Fruits & Toxique, utilisé pour piéger les voleurs de vin de Raphia & + \\
\hline Ficus trichopoda & Moraceae & 0,75 & 35 & Ecorces & $\begin{array}{l}\text { Médicinal: En association avec l'écorce de Syzygium } \\
\text { owariense: tisane pour nouveau-né }\end{array}$ & +++ \\
\hline Hallea ledermanni & Rubiaceae & 0,32 & 7,6 & Bois & Bois d'œuvre et de service & ++++ \\
\hline Laccosperma opacum & Arecaceae & - & 7,70 & Feuilles & Fabrication de nasses et meubles & ++ \\
\hline Mikania carteri & Asteraceae & - & 58 & Feuilles & Médicinal: contre vomissements & + \\
\hline Nauclea diderrichii & Rubiaceae & 2 & 54 & Ecorces & $\begin{array}{l}\text { Médicinal: écorce contre constipation, maux de ventre, } \\
\text { règles douloureuses, ulcère }\end{array}$ & ++++ \\
\hline Psychotria articulata & Rubiaceae & 0,015 & 11,54 & Tiges & Bois résistant, charpente & +++ \\
\hline Raphia hookeri & Arecaceae & 3,85 & 81 & $\begin{array}{l}\text { Feuilles, } \\
\text { Stipe }\end{array}$ & $\begin{array}{l}\text { Grenier, plafond, exploitation du vin, meubles, fibres, } \\
\text { nasses, cordes }\end{array}$ & ++++ \\
\hline Smilax kraussiana & Smilacaceae & - & 35 & Tiges & Corde pour divers usages & + \\
\hline Sorindeia warneckei & Anacardiaceae & - & 15,40 & $\begin{array}{l}\text { Feuilles et } \\
\text { racines }\end{array}$ & Médicinal: contre la dysenterie, ulcère & + \\
\hline Spondianthus preussii & Euphorbiaceae & 1,31 & 9,64 & Tiges & Charpente, plafond & +++ \\
\hline Syzygium owariense & Myrtaceae & 6 & 77 & Ecorces & Médicinal: tisane pour nouveau-né & +++ \\
\hline Xylopia aethiopica & Annonaceae & 0,0065 & 3,84 & Fruits & Médicinal: contre l'ulcère & + \\
\hline Xylopia rubescens & Annonaceae & 11 & 100 & Bois & Pirogues et plafond & ++ \\
\hline
\end{tabular}



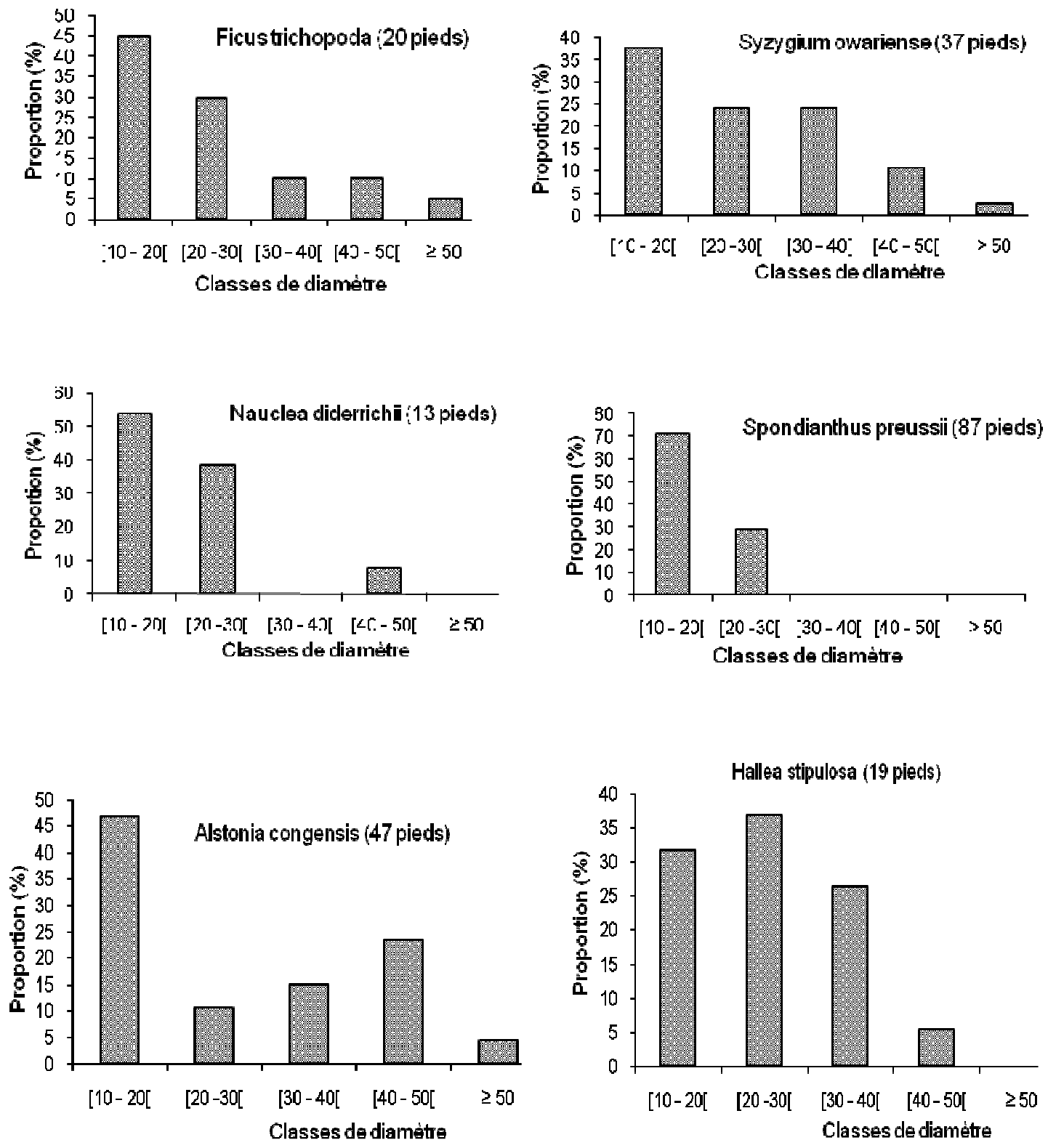

Figure 4: Structure diamétrique des espèces d'arbre prisées par la population locale.

Selon White $(1979$, 1986), la forêt marécageuse de Lokoli est située dans le couloir sec dahoméen (ou Dahomey Gap), plus précisément dans la zone régionale de transition Guinéo-Congolaise/Soudanienne. Mais, la présente étude montre que cette forêt abrite beaucoup d'espèces caractéristiques des forêts marécageuses Guinéo-Congolaises dont Hallea ledermannii, Uapaca paludosa, Syzygium owariense, Xylopia rubescens, Anthostema aubryanum, Rothmannia munsae, Spondianthus preussii et Carapa procera. La nature guinéo-congolaise de la forêt marécageuse de Lokoli est confirmée par l'analyse phytogéographique qui montre que plus de $70 \%$ des espèces recensées sont Guinéo-Congolaises (Adomou et al., 2007). Ainsi, bien que le Dahomey Gap crée une discontinuité dans la "ceinture" forestière ouest-africaine, elle ne constitue pas une barrière infranchissable aux espèces végétales (Jongkind et Hawthorne, 2005).

La présence des espèces de forêt dense humide semi-décidue telles que Rothmannnia longiflora, Diospyros abyssinica et Cola gigantea est due à la présence de buttes sablo- 
limoneuses qui jouent un rôle de microstations contribuant ainsi à l'augmentation de la diversité spécifique.

La richesse spécifique (125 espèces) dans la forêt de Lokoli se situe dans la marge de 100-150 espèces fixée par Sokpon et al. (2001) pour les forêts marécageuses du Bénin. La forêt marécageuse de Lokoli apparaît plus riche que celles de Mondo-Tokpa (68 espèces) et de Sèmè-Kpodji (67 espèces; Paradis, 1975) qui sont très dégradées. Seulement 22 et 45 espèces végétales ont été recensées par Akoègninou (2004) dans les forêts marécageuses à Alstonia congensis et Psychotria articulata de Sèmè $\left(6^{\circ} 23^{\prime} \mathrm{N}, 2^{\circ} 37^{\prime}\right.$ E) et de Igolo $\left(6^{\circ} 38^{\prime} \mathrm{N}, 2^{\circ} 44^{\prime} \mathrm{E}\right)$ à l'est du Bénin, lesquelles se trouvent aujourd'hui dans un état de dégradation poussée. Mais cette valeur est inférieure à celle trouvée par Dengueadhe Kolongo (1999) dans la forêt marécageuse de Taï en Côte d'Ivoire (187 espèces) où plusieurs plantes endémiques au bastion forestier occidental sont recensées (Bongers et al., 2004).

Du fait de la forte sélectivité du milieu marécageux, on y note une faible richesse spécifique et une mauvaise distribution des abondances. Ceci se traduit dans la forêt de Lokoli par une faible équitabilité de Piélou $(0,63)$ lorsqu'on considère les individus de $\mathrm{dbh} \geq 10 \mathrm{~cm}$. Aussi, les espèces écologiquement dominantes par leur dispersion, dbh et densité sont représentées par huit espèces (tableau 1) qui occupent plus de $80 \%$ de la valeur d'importance totale des espèces ligneuses.

La valeur élevée de la densité (620 tiges/ha) s'explique par le fait que les troués sont rapidement colonisées par Raphia hookeri, dont la contribution est évaluée à $17 \%$ sur l'ensemble de la forêt.

La forêt de Lokoli abrite une trentaine d'espèces végétales menacées de disparition au Bénin (Adomou, 2005). Certaines espèces répertoriées dans cet écosystème se révèlent d'un grand intérêt pour la conservation de la biodiversité végétale au Bénin. Trois catégories d'espèces de plantes sont distinguées (Hawthorne, 1996; Poorter et al., 2004; Adomou, 2005):

* Espèces à large distribution en Afrique mais rares au Bénin. Il s'agit de: Crudia senegalense (Leguminosae), Cuviera macroura (Rubiaceae), Ficus lyrata (Moraceae), Rothmannia munsae (Rubiaceae) et Syzygium owariense (Myrtaceae).

* Espèces endémiques Guinéo-Congolaises rares en Afrique. Il s'agit des espèces telles que: Xylopia rubescens (Annonaceae) et Uapaca paludosa (Euphorbiaceae).

* Espèces menacées de disparition en Afrique, parmi lesquelles on peut citer: Laccosperma opacum (Arecaceae), Hallea ledermannii (Rubiaceae), Nauclea diderrichii (Rubiaceae), Pycnanthus angolensis (Myristicaceae) et Xylopia aethiopica (Annonaceae).

La structure diamétrique du peuplement ligneux montre que la dynamique de la forêt serait influencée par la pression anthropique. Les faciès dégradés sont caractérisés par une très faible fréquence des grandes classes de diamètre $(>40 \mathrm{~cm})$ et une forte densité de Raphia hookeri. Dans les années 2000, des exploitants nigérians auraient abattus un nombre important de gros sujets d'arbres dont certains sont abandonnés suite à un affrontement avec la population locale qui aurait énergiquement répliqué. Ceci semble affecter la structure démographique des espèces comme Alstonia congensis et Hallea stipulosa (figure 4) dont les bois d'œuvre sont très recherchés.

La pression sur Spondianthus preussii est sélective et porte sur les tiges de faible diamètre $(10-20 \mathrm{~cm})$, les perches étant utilisées pour confectionner la charpente des toits. Cependant, l'ensemble du peuplement ligneux tend à afficher une structure en "J" (figure 3) caractéristique de la maturation et de la stabilisation de la forêt. Ainsi, l'abondance des tiges de faible diamètre (10$20 \mathrm{~cm}$ ) suppose l'existence de conditions favorables à la régénération naturelle des espèces.

La sensibilité des espèces à l'écorçage différerait d'une espèce à une autre (Miller et al., 2007). Ainsi, La structure diamétrique de Nauclea diderrichii est plus affectée par l'écorçage que celles de Ficus trichopoda et Syzygium owariense, en supposant que ces espèces ne sont pas exploitées à d'autres fins. Les études récentes ont montré que l'écorçage affecte négativement aussi bien la croissance végétative que la performance reproductive des arbres (Scogings et Macanda, 2005). La population devra donc être sensibilisée aux techniques appropriées de prélèvement des écorces pour garantir une utilisation durable des ressources.

\section{Conclusion}

La forêt de Lokoli apparaît comme un des rares écosystèmes marécageux les mieux conservés dans les zones humides du Bénin. 
Avec son cortège floristique et faunique unique dans le contexte béninois, cette forêt est bien indiquée pour la conservation de la biodiversité au Bénin. Environ $85 \%$ des espèces végétales répertoriées se révèlent génériquement distincts, ce qui traduit une grande diversité taxonomique. La forêt est d'une grande utilité pour la population locale qui l'a préservée depuis des siècles. L'Etat béninois a la responsabilité de les assister pour garantir une conservation et une gestion durables.

\section{REFERENCES BIBLIOGRAPHIQUES}

Adomou AC. 2005. Vegetation patterns and environmental gradient in Benin: Implications for biogeography and conservation. $\mathrm{PhD}$ thesis, Wageningen University, the Netherlands, 136.

Adomou AC, Akoègninou A, Sinsin B, De Foucault B, Van der Maesen LJG. 2007. Biogeographical analysis of the vegetation in Benin. Acta Bot. Gallica, 154(2): 221-233.

Akoègninou A 2004. Recherches botaniques et écologiques sur les forêts actuelles du Bénin. Thèse d'Etat, Université de Cocody-Abidjan, 326p.

Akoègninou $\mathrm{A}$, van der Burg $\mathrm{WJ}$, van der Maesen LJG 2006. Flore Analytique du Bénin. Backhuys Publishers: Wageningen; 1034p.

Bongers F, Poorter L and Hawthorne WD 2004. The forest of Upper Guinea: gradients in large species composition. In Biodiversity of West African Forests: An ecological atlas of woody plants species, Poorter L, Bongers F, Kouamé FN, Hawthorne WD (eds). CABI Publishing: Oxon, UK and Cambridge; 4: 41-52.

Braun-Blanquet J 1932. Plant Sociology: The Study of Plant Communities. Ed. Mc Gray Hill: London, United Kingdom; $349 \mathrm{p}$.

Curtis JT, Macintosh RP. 1950. The interrelations of certain analytic and synthetic phytosociological characters. Ecology, 31(3): 435-455.

Dengueadhe Kolongo TS. 1999. Etude de la végétation du Sud du Parc National de Taï : identification des milieux. Rapport, Lab. de Botanique, Univ. de Cocody, Côte d'Ivoire.

Frontier S. 1983. Choix et contraintes de l'échantillonnage écologique. In Stratégie d'Echantillonnage en
Ecologie, Frontier S (éd.). Les Presses de l'Univ. de Laval: Laval; 3-62.

Frontier S, Pichod-Viale D. 1991. Ecosystème: Structure, Fonctionnement, Evolution. Collection d'Ecologie. Masson: Paris; 392p.

Hawthorne WD, Jongkind C. 2006. Woody plants of Western African Forests: A Guide to the Forest Trees, Shrubs and Lianes from Senegal to Ghana. Kew Publishing: Royal Botanic Gardens: Kew; 1023.

Hawthorne WD. 1996. Holes and the sums of parts in Ghanaian forest: regeneration, scale and sustainable use. Proceedings of the Royal Society of Edinburgh, 104B: 75-176.

Jongkind C, Hawthorne WD. 2005. A botanica synopsis of the lianes and other forest climbers. In Forest Climbing Plants of West Africa: Diversity, Ecology and Management, Bongers F, Parren MPE and Traoré D (eds). CAB International: UK and Cambridge; 19-39.

INSAE 2002. Troisième recensement général de la population et de l'habitat. Résultats définitifs: Tableaux statistiques Cotonou, Bénin, 4p.

Lebrun J, Gilbert G. 1954. Une classification écologique des forêts du Congo. Publ. I.N.E.A.C., sér. Sc., 63: 89.

Mangenot G. 1956. Etude sur la forêt des plaines et des plateaux littoraux de la Côte d'Ivoire. Etudes Eburnéennes, I.F.A.N., Dakar, Tome 4: 5-61.

Miller RE, Harry WA, Reukema DL, Max TA. 2007. Growth of Bear-Damaged Trees in a Mixed Plantation of Douglasfir and Red Alder. Research Paper PNWRP-571, USDA: 40p.

Mondjannagni A. 1969. Contribution à l'étude des paysages végétaux du Bas-Dahomey. Ann. Univ. Abidjan. Sér. G., 1(2): 191p.

Mori SA, Boom BM. 1987. The Lecythidaceae of a lowland Neotropical forest. New York Bot. Gard., 44: 9-29.

Ourab N, Achir M, Khettal N, Goumiri K, Smara Y. 2003. Application à l'analyse multirésolution et des méthodes floues pour la fusion et la classification des images satellitaires. Télédétection, 3(1): 17-31

Palatucci M, Mitchell TM. 2007. Classification in Very High Dimensional Problems with Handfuls of Examples, Principles and Practice of Knowledge 
Discovery in Databases. SpringerVerlag.

Paradis G. 1975. Physionomie, composition floristique, et dynamisme des formations de la basse vallée de l'Ouémé (Dahomey). Ann. Univ. Abidjan Série C., 11: 35-101.

PAZH (Programme d'Aménagement des Zones Humides). 1997. Rapport de formulation. $\mathrm{ABE} / \mathrm{CBDD} / \mathrm{Cotonou} / \mathrm{R}$. Bénin, 63p.

Poorter L, Bongers F, N'Kouamé F, Hawthorne WD. 2004. Biodiversity of West African Forests: an Ecological Atlas of Woody Plants Species. CABI Publishing: Cambridge, USA.

Reitsma JM. 1988. Forest Vegetation in Gabon. Tropenbos Technical series 1. Tropenbos Foundation: The Netherlands; $142 \mathrm{p}$.

Scogings P, Macanda M. 2005. Acacia karroo responses to early dormant season defoliation and debarking by goats in a semi-arid subtropical savanna. Plant Ecology, 179(2): 193-206.

Shannon CE. 1948. A mathematical theory of communications. Bell Syst. Techn. J., 27: 623-656.

Sinsin B, Assogbadjo EA. 2002. Diversité, structure et comportement des primates de la forêt marécageuse de Lokoli au Bénin. Biogeographica, 78(4): 129-140.

Sokpon N, Sinadouwirou T, Gbaguidi F, Biaou H. 2001. Aperçu sur les forêts édaphiques hygrophiles du Bénin. Belg. Journ. Bot., 134(1): 79-93.

White F 1979. The Guineo-Congolian Region and its relationships to other phytochoria. Bull. Jard. Bot. Belg., 49: 11-55.

White F 1986. La Végétation de l'Afrique. ORSTOM-UNESCO; 87p. 